Check for updates

Cite this: RSC Adv., 2018, 8, 22392

\title{
Sulfonated covalent triazine-based frameworks as catalysts for the hydrolysis of cellobiose to glucose $\uparrow$
}

\author{
Jens Artz, (D) ${ }^{a}$ Irina Delidovich, (D) ${ }^{a}$ Moritz Pilaski, ${ }^{a}$ Johannes Niemeier, (D)
} Britta Maria Kübber, ${ }^{a}$ Khosrow Rahimi ${ }^{b}$ and Regina Palkovits (D) *a

Covalent triazine-based frameworks (CTFs) were synthesized in large scale from various monomers. The materials were post-synthetically modified with acid functionalities via gas-phase sulfonation. Acid capacities of up to $0.83 \mathrm{mmol} \mathrm{g}^{-1}$ at sulfonation degrees of up to $10.7 \mathrm{~mol} \%$ were achieved. Sulfonated CTFs exhibit high specific surface area and porosity as well as excellent thermal stability under aerobic conditions $\left(>300^{\circ} \mathrm{C}\right)$. Successful functionalization was verified investigating catalytic activity in the acidcatalyzed hydrolysis of cellobiose to glucose at $150{ }^{\circ} \mathrm{C}$ in $\mathrm{H}_{2} \mathrm{O}$. Catalytic activity is mostly affected by porosity, indicating that mesoporosity is beneficial for hydrolysis of cellobiose. Like other sulfonated materials, S-CTFs show low stability under hydrothermal reaction conditions. Recycling of the catalyst is challenging and significant amounts of sulfur leached out of the materials. Nevertheless, gas-phase sulfonation opens a path to tailored solid acids for application in various reactions. S-CTFs form the basis for multi-functional catalysts, containing basic coordination sites for metal catalysts, tunable structural parameters and surface acidity within one sole system.

Received 18th May 2018

Accepted 12th June 2018

DOI: $10.1039 / \mathrm{c} 8 \mathrm{ra0} 4254 \mathrm{c}$

rsc.li/rsc-advances diffusion limitation and thus, comparably low catalytic activity. ${ }^{6}$ To overcome such pore diffusion limitations, the sulfonation of macroporous polymers has been studied intensively. In this regard, especially polystyrene-based materials gained a lot of attention. ${ }^{8}$ Unfortunately, thermal stability in comparison to zeolites is relatively low and desulfonation takes place at 260$300{ }^{\circ} \mathrm{C}$ in the gas or at $120-150{ }^{\circ} \mathrm{C}$ in the liquid phase. ${ }^{9,10}$ Due to their ecological balance, solid acids are often used in "green" chemical processes, for example in the production of biodiesel. ${ }^{\mathbf{1 1} 12}$ Solid acids can be used as catalysts for both the transesterification of triglyceride in the classical bio-diesel production as well as in the hydrolysis of biomass to desired bio-diesel-like compounds. ${ }^{12-15}$

In this respect, hydrolysis of water-insoluble cellulose to soluble glucose attracts great attention as the most challenging step for the production of bio-ethanol, bio-HMF and other value-added products. ${ }^{16}$ Herein, we focus on a soluble dimer of glucose, cellobiose, representing a structural unit of cellulose. Vilcocq et al. has recently reviewed the activity of different solid catalysts for hydrolysis of cellobiose into glucose. ${ }^{17}$ Inorganic materials, such as zeolites ${ }^{17}$ or metal oxides, ${ }^{18}$ exhibited moderate catalytic activity and selectivity for glucose formation. The best catalytic performance was demontrated by sulfonated materials, including resins ${ }^{19}$ carbons $^{20}$ silicas $^{21}$ or organosilicas: ${ }^{22}$ up to quantitative yield of glucose can be attainable over these catalysts. However, stability of the materials against leaching of sulfur under reaction conditions appears to be the main challenge for reusability of the catalysts. ${ }^{21-23}$ At the same 
time, other structural changes of the materials were reported. For example, Zhao et al. observed elimination of oxygencontaining groups from the surface of the sulfonated graphite oxide used as catalyst for cellobiose hydrolysis. ${ }^{24}$ Therefore, water-tolerant acidic materials are of great interest for the hydrolysis reaction.

In this regard, covalent triazine-based frameworks (CTFs; Scheme 1) fulfil all necessary requirements for a solid acidic catalyst resulting after sulfonation. ${ }^{25,26}$ Thermally stable up to $400{ }^{\circ} \mathrm{C}$ and chemically robust under relatively harsh reaction conditions, these materials have drawn the interest of many researchers as solid catalyst supports. ${ }^{27-29}$ Additionally, they possess sufficient benzylic structure elements for a feasible sulfonation, while access to a large variety of commercial dinitrile monomers grants control of the resulting materials porosities and specific surface areas. ${ }^{29}$

The sulfonation of aromatic systems is an electrophilic aromatic substitution which in general occurs in the liquid phase in presence of oleum or highly concentrated $\mathrm{H}_{2} \mathrm{SO}_{4}{ }^{30-32}$ To perform sulfonation reactions of polymeric materials under milder conditions, the treatment of a solid sample with $\mathrm{SO}_{3}$ in the gas-phase is possible. ${ }^{3,34}$ In this manner, sole surface adsorption of the acid groups can be suppressed, while covalent bonding becomes favoured. First attempts to implement sulfuric species within CTF materials were described by Bai et $a l .{ }^{35}$ Utilizing thiourea and thiosemicarbazide during a polymerization step with cyanuric chloride resulted in sulfur containing CTF materials with high sorption capacities and selectivities for uranium(vi). More recently, Talapaneni et al. substituted $\mathrm{ZnCl}_{2}$ by elemental polymeric sulfur to enable a solvent and catalyst-free polymerization of 1,4-dicyanobenzene to the corresponding CTF. ${ }^{36}$ This way, a homogeneous distribution of sulfur at high contents of $62 \mathrm{wt} \%$ was achieved. The resulting material exhibited excellent properties as a cathode material in $\mathrm{Li}-\mathrm{S}$ batteries with superior initial<smiles>N#Cc1cccc(C#N)c1</smiles><smiles>N#Cc1cccc(C#N)n1</smiles><smiles>N#Cc1ccc(C#N)cc1</smiles>

1,3-DCB (CTF-a)

2,6-DCP (CTF-b)

1,4-DCB (CTF-C)

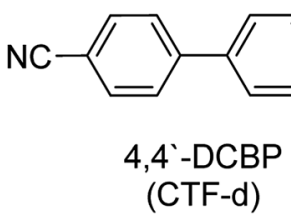

Scheme 2 Monomers applied in the CTF preparation.

coulombic efficiencies and cycle lifetimes. However, the porous character of the materials was entirely lost.

Herein, we present a novel approach to obtain solid acid catalysts with controllable structural properties based on CTFs. CTFs obtained from a variety of dinitrile monomers (Scheme 2) have been synthesized in large scale, resulting in materials with the desired structural properties, namely porosity, specific surface area and nitrogen content. The influence of these structural properties on the gas-phase sulfonation method has been studied intensively. Subsequently, the catalytic activity in the hydrolysis reaction of cellobiose to glucose was investigated with regard to the catalysts acidity, porosity and specific surface area.

\section{Experimental section}

\section{Preparation of the CTF materials}

For the synthesis of the CTF-a material, 1,3-dicyanobenzene (3.00 g, $23.4 \mathrm{mmol}, 1$ eq.) and $\mathrm{ZnCl}_{2}$ (15.96 g, $117.1 \mathrm{mmol}, 5$ eq.)
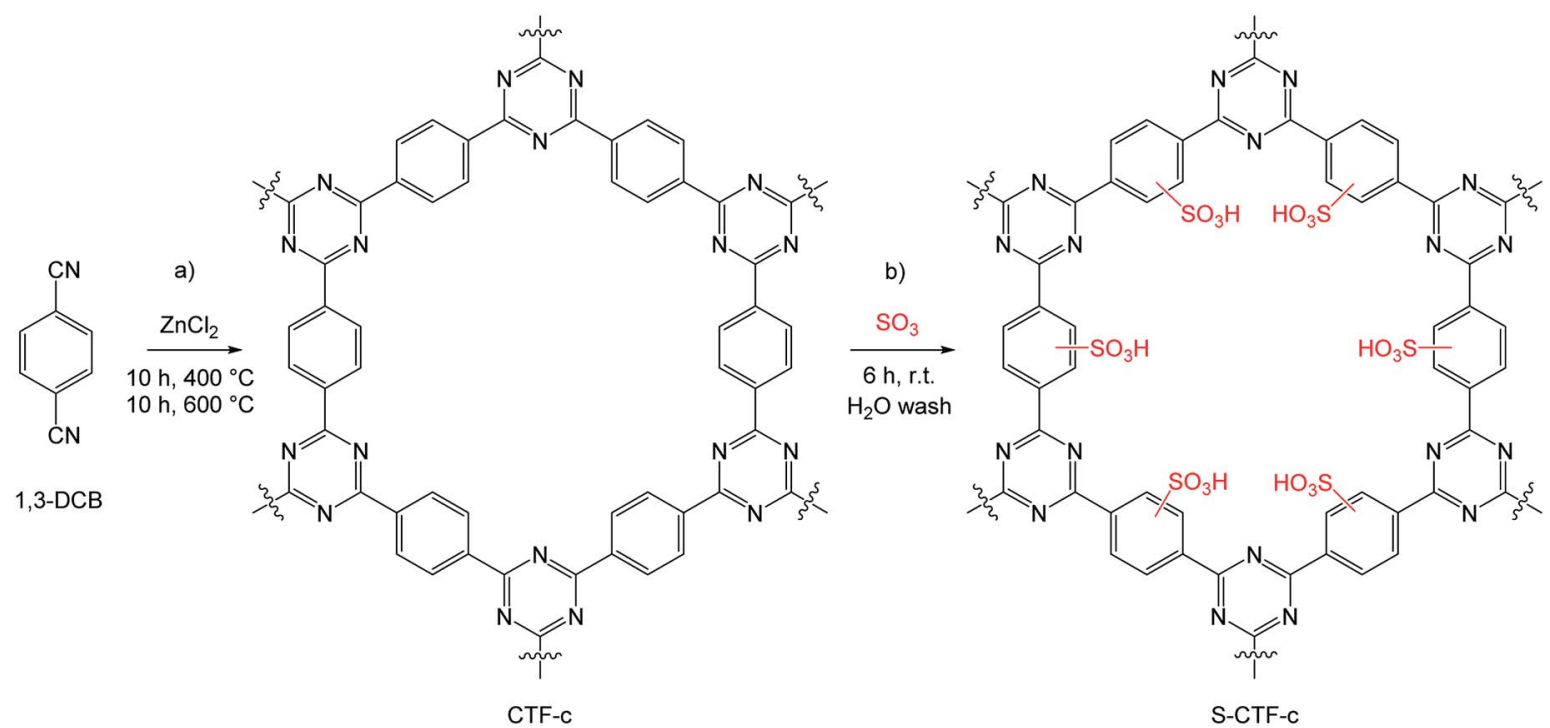

Scheme 1 (a) Idealized synthesis of a CTF based on 1,4-DCB as a monomer (CTF-c). (b) Proposed sulfonation methodology resulting in the sulfonated CTF material (S-CTF-C). 
were mixed and ground together within a glovebox, transferred into a quartz ampule (12 cm height and $3 \mathrm{~cm}$ diameter) and dried in vacuum for at least 3 hours. The ampule was then flame-sealed and placed inside a furnace for 10 hours of heat treatment at $400{ }^{\circ} \mathrm{C}$ and further 10 hours at $600{ }^{\circ} \mathrm{C}$ (heating rate: $10 \mathrm{~K} \mathrm{~min}^{-1}$ ). After cooling to room temperature, the ampule was broken open (CAUTION: the ampules are under pressure, which is released during opening) and the solid product was ground and washed thoroughly with water and diluted $\mathrm{HCl}(0.1 \mathrm{M})$. The solid material was then ground in a ball mill (Fritsch Pulverisette $23,5 \mathrm{~min}, 30 \mathrm{~Hz}$ ) to obtain a black powder, which was finally washed successively with water, diluted $\mathrm{HCl}$, diluted $\mathrm{NaOH}$, water and THF. Afterwards, it was dried in vacuum for at least $12 \mathrm{~h}$ at $60{ }^{\circ} \mathrm{C}$. Materials based on 2,6-pyridinedicarbonitrile (CTF-b), 1,4-dicyanobenzene (CTF-c) and 4,4'-biphenyldicarbonitrile (CTF-d) were synthesized as described for 1,3-DCB ( $3.00 \mathrm{~g}$ of monomer, $5 \mathrm{eq}$. of $\mathrm{ZnCl}_{2}$ ). As the filling degree of the ampule apparently has no effect on the physical parameters of the resulting polymer, the ampule was charged to a maximum of half ampule volume to prevent bursting within the furnace.

\section{Gas-phase sulfonation of the materials}

Gas-phase sulfonation reactions of all materials have been carried out in a glass reactor equipped with a glass frit according to literature (Fig. S8 $\dagger$ ). ${ }^{34}$ After evacuation of the sample for at least $30 \mathrm{~min}$ (tap 1 closed), the CTF material (1.0 g) is exposed to $\mathrm{SO}_{3}$ gas (tap 1 opened). The $\mathrm{SO}_{3}$ gas is withdrawn from a reservoir (equipped with a stirring bar, $300 \mathrm{rpm}$ ) filled with $20 \%$ oleum $(10 \mathrm{~mL})$ via dynamic vacuum ( $p<0.035 \mathrm{mbar})$. Within the reactor, the $\mathrm{SO}_{3}$ reacts with the CTF sample at ambient temperature. After $6 \mathrm{~h}$ tap 1 is closed and vacuum is applied to the sulfonated sample for further $30 \mathrm{~min}$. The material is suspended carefully in water, filtered and washed thoroughly with water until a neutral $\mathrm{pH}$ value of the washing solution is attained. The S-CTF sample is then dried within a furnace (at $80^{\circ} \mathrm{C}$, ambient pressure) and a vacuum furnace (at $60{ }^{\circ} \mathrm{C}$ ) for at least $12 \mathrm{~h}$.

\section{Hydrolysis of cellobiose to glucose with S-CTF}

In a typical experiment cellobiose (0.5 g, $1.5 \mathrm{mmol})$, catalyst $(0.05 \mathrm{~g})$ and $4.5 \mathrm{~mL}$ water were charged into a stainless steel autoclave with a gas inlet. The autoclave was sealed, pressurized to 30 bar of nitrogen and heated to $110-150{ }^{\circ} \mathrm{C}$ under stirring at $750 \mathrm{rpm}$. After the experiment, the autoclave was cooled down in an ice bath and depressurized. The catalyst was filtered off using a syringe filter (CHROMAFIL® Xtra, PA-20/25, $0.20 \mu \mathrm{m}$ ) and the reaction solution was analyzed via HPLC (Shimadzu 2020) using two successfully connected organic acid resin columns $(100 \mathrm{~mm} \times 8.0 \mathrm{~mm}$ and $300 \times 8.0 \mathrm{~mm})$ as previously reported. ${ }^{45}$ For recycling studies (after $2 \mathrm{~h}$ reaction time) the catalyst $(0.075 \mathrm{mg})$ was filtered off via a Whatman ${ }^{\mathrm{TM}}$ filtration system equipped with Anodisc $25(0.20 \mu \mathrm{m})$ membranes, thoroughly washed with water and dried overnight in vacuum at $60{ }^{\circ} \mathrm{C}$.

\section{Characterization of the CTF and S-CTF materials}

CHNS elemental analysis was performed on a PerkinElmer ${ }^{\circledR}$ 2400 Series II CHNS/O Elemental Analyzer and supported by measurements carried out at the microanalytical laboratory Kolbe (Mülheim an der Ruhr, Germany). ICP-OES analysis was conducted with a SPEKTROFLAME ICP-D instrument from SPEKTRO-Analytical Instruments. The samples $(0.03 \mathrm{~g})$ were molten in the presence of $\mathrm{KNO}_{3}(0.12 \mathrm{~g})$ and $\mathrm{KOH}(1 \mathrm{~g})$ within a porcelain crucible and dissolved in aqueous $\mathrm{HCl}$. For titration of the sulfonated CTFs, samples $(0.05 \mathrm{~g})$ were dispersed in $10 \mathrm{~mL}$ of saturated $\mathrm{NaCl}$ solution (aq.) and stirred (500 rpm) for 1 week before titration to guarantee complete ion exchange. The titrations were then carried out using a HI $9124 \mathrm{pH}$-meter from HANNA INSTRUMENTS and $0.005 \mathrm{M} \mathrm{NaOH}$ (aq.). Thermogravimetric analysis (TGA) was carried out in air $\left(60 \mathrm{~mL} \mathrm{~min}^{-1}\right)$ on a Netzsch Simultaneous Thermal Analyzer Type STA449G Jupiter instrument at a heating rate of $10 \mathrm{~K} \mathrm{~min}^{-1}$. Nitrogen physisorption experiments were conducted on a Micromeritics ASAP 2010 instrument. Samples were degassed for at least $15 \mathrm{~h}$ at $150{ }^{\circ} \mathrm{C}$ using a FloVacDegasser. Static volumetric measurements were carried out at $-195.8^{\circ} \mathrm{C}$. The empty volume of the cell was determined with helium. The specific surface area was determined with the Brunauer-Emmet-Teller method (BET) using data points at a relative pressure $p / p_{0}$ between 0.05 and 0.3 . The total pore volume was determined at a relative pressure of 0.98 . The pore size distribution was calculated via MicroActive (version 1.01) using the density functional theory (DFT) $\mathrm{N}_{2}$-model for slit geometry at optimal Goodness of Fit $v s$. Regularization (0.01) values for both RMS Error of Fit and Roughness of Distribution. The cumulative pore volume at the pore width of $2 \mathrm{~nm}$ was used to determine the micropore volume of the samples. Scanning electron microscopy (SEM) images were recorded on a Hitachi SU9000 Ultra-high resolution microscope equipped with a cooled $\mathrm{Si}(\mathrm{Li})$ X-ray detector Oxford Inca. The acceleration voltage was set to $20 \mathrm{kV}$. SEM samples were prepared by adsorbing dry powder on a $\mathrm{Cu} /$ lacey carbon grid and shaking off any loose material. Transmission electron microscopy (TEM) images were recorded on a Zeiss Libra 120 microscope. TEM samples were prepared by adsorbing dry powder on a $\mathrm{Cu}$ /lacey carbon grid and shaking off any loose material.

\section{Results and discussion}

\section{CTF preparation}

The synthesis of various CTF materials with different structural properties was conducted using 1,3-dicyanobenzene (1,3-DCB), 2,6-dicyanopyridine (2,6-DCP), 1,4-dicyanobenzene (1,4-DCB) and $4,4^{\prime}$-biphenyldicarbonitrile $\left(4,4^{\prime}\right.$-DCBP $)$ as monomers (Scheme 2). As described elsewhere, bimodal microporous and mesoporous materials containing numerous $\mathrm{N}$ moieties are accessible via ionothermal synthesis in molten $\mathrm{ZnCl}_{2}$ acting as a solvent and a Lewis-acidic catalyst. ${ }^{25}$ Since $\mathrm{ZnCl}_{2}$ furthermore acts as a porogen, an excess $\mathrm{ZnCl}_{2} /$ monomer molar ratio of $5: 1$ was applied. Sequential heating of the monomer/salt mixture for $10 \mathrm{~h}$ each to 400 and $600{ }^{\circ} \mathrm{C}$ in quartz ampules leads to the 
formation of an amorphous and partially carbonized framework containing tunable amounts of $\mathrm{N}$ with exceptionally high porosity and specific surface areas.

In prior studies, quartz ampules of small volumes $(l=12 \mathrm{~cm}$, $\varnothing=1.5 \mathrm{~cm}$ ) have been used to guarantee sufficient heat transfer during the polymerization reaction. ${ }^{29}$ Unfortunately, the small ampule volume restricts to small quantities of the desired material and therefore the synthesis becomes both cost and time intensive. To tackle this challenge, ampules of larger volume $(l=$ $12 \mathrm{~cm}, \varnothing=3.0 \mathrm{~cm}$ ) were used in the present study and result in approximately the 5 -fold quantity of the desired product (4-fold ampule volume increase combined with increasing filling height). This enabled increasing the CTF yield from $0.6 \mathrm{~g}$ to approximately $3.0 \mathrm{~g}$ per batch. Properties of the CTFs synthesized in small and large ampules are summarized in Table 1 . The resulting CTF materials were investigated via $\mathrm{N}_{2}$ physisorption as well as elemental and thermogravimetric analysis (Table 1, Fig. S1-S5 $\dagger$ ). The materials obtained show the characteristic $\mathrm{N}_{2}$ physisorption isotherms comparable to the previously described CTFs synthesized in small scale. ${ }^{29}$ In this regard, $\mathrm{N}_{2}$ physisorption isotherms of CTFs based on 1,3-DCB (CTF-a, Fig. S1 $\dagger$ ), 1,4-DCB (CTF-c, Fig. S3 $\dagger$ ) and 4,4'-DCBP (CTF-d, Fig. S4 $\dagger$ ) correspond to type IV isotherms, emphasizing the mesoporous structure of all materials. While the hysteresis for both CTF-a and -c is barely existent, CTF-d exhibits a strongly pronounced hysteresis according to the significantly increased porosity, as it would be expected from the structural properties of the monomer. Regardless of the synthesis scale, a material based on 2,6DCP (CTF-b, Fig. S2 $\dagger$ ) presents a type I isotherm characteristic for completely microporous materials. The most drastic decrease in porosity and specific surface area when applying large scale synthesis is found for CTF-a based on 1,3-DCB (Table 1, entry 1 and 2). This might be correlated to the incontrollable orientation of the meta-substituted nitrile-function during polymerization, thus leading to unregulated structural parameters. In contrast, only a slight decrease in specific surface area and microporosity is observed for CTF-b (Table 1, entry 4 and 5). This can be assigned to the dense coordination sphere during polymerization, which is caused by coordination of the pyridine backbone to the Lewis-acidic $\mathrm{ZnCl}_{2}$ regardless of the synthesis scale. A similar trend can be observed for CTF-c based on 1,4-DCB, which exhibits a nearly unchanged specific surface area and pore volume regardless of the production scale (Table 1, entry 8 and 9). Interestingly, the specific surface area for CTF-d increases while the total pore volume decreases slightly in large scale synthesis, which can be assigned to favored partial carbonization as reflected by the lower amount of $\mathrm{N}$ within the resulting polymer (Table 1, entry 11 and 12). In both cases, the rigid structure of the para-substituted monomers seems to play an important role during the formation of the porous framework. All four materials have been synthesized repeatedly to investigate the reproducibility of the large scale synthesis method (Table S1 and Fig. S7†). Only minor changes in specific surface areas and total pore volume could be detected and the course of the isotherms was unchanged.

The $\mathrm{N}$ content of the resulting CTFs is slightly lower for all materials synthesized in larger scale, except for CTF-b. This seems to correspond to a slightly favored carbonization under these reaction conditions. Nevertheless, reproducibility tests for material synthesis (Table S1 $\dagger$ ) delivered comparable results for CHN-analysis, confirming the $\mathrm{N}$ contents reported in prior studies. ${ }^{29}$ In summary, $\mathrm{N}$ contents within the resulting materials follow the same trend as reported for small scale ampule synthesis and can be correlated to the amount of nitrogen available in the used monomers with slight deviations due to partial carbonization.

\section{Sulfonation in the gas-phase}

Via sulfonation, acid sites can be introduced into the solid material, resulting in an acidic catalyst. By introducing acid sites, both polarity and hence hydrophilicity will be increased.

Table $1 \mathrm{CHN}$ elemental analysis, specific surface area, total pore volume of untreated and sulfonated CTF materials as well as the sulfur content after modification with $\mathrm{SO}_{3}$ in the gas-phase

\begin{tabular}{|c|c|c|c|c|c|c|c|}
\hline No. & Material & $\mathrm{C}^{a}[\mathrm{wt} \%]$ & $\mathrm{H}^{a}[\mathrm{wt} \%]$ & $\mathrm{N}^{a}[\mathrm{wt} \%]$ & $S_{\mathrm{BET}}^{b}\left[\mathrm{~m}^{2} \mathrm{~g}^{-1}\right]$ & $V_{\mathrm{P}} / V_{\mathrm{P}(\text { micro })}{ }^{c}\left[\mathrm{~cm}^{3} \mathrm{~g}^{-1}\right]$ & $\mathrm{S}^{a, d}[\mathrm{wt} \%]$ \\
\hline 1 & CTF-a ${ }^{e}$ & 72.7 & 2.8 & 9.5 & 2439 & $1.96 / 0.47$ & - \\
\hline 2 & CTF-a & 71.2 & 2.2 & 7.9 & 1840 & $1.15 / 0.44$ & - \\
\hline 4 & $\mathrm{CTF}-\mathrm{b}^{e}$ & 48.8 & 3.9 & 17.2 & 1179 & $0.64 / 0.64$ & - \\
\hline 5 & CTF-b & 59.1 & 3.1 & 17.6 & 972 & $0.54 / 0.38$ & - \\
\hline 6 & S-CTF-b & - & - & - & 603 & $0.33 / 0.24$ & 2.67 \\
\hline 9 & CTF-c & 62.1 & 2.5 & 6.6 & 2001 & $1.37 / 0.44$ & - \\
\hline 10 & S-CTF-c & - & - & - & 1206 & $0.65 / 0.37$ & 1.27 \\
\hline 11 & CTF-d ${ }^{e}$ & 84.9 & 1.7 & 3.7 & 1683 & $2.63 / 0.30$ & - \\
\hline 12 & CTF-d & 75.5 & 4.3 & 1.3 & 1859 & $1.54 / 0.30$ & - \\
\hline 13 & S-CTF-d & - & - & - & 1561 & $1.24 / 0.26$ & 1.57 \\
\hline
\end{tabular}

${ }^{a}$ Determined with elemental analysis. ${ }^{b}$ Specific surface area identified by Brunauer-Emmet-Teller (BET) method. ${ }^{c}$ Total pore volume determined at $p / p_{0}=0.98$ and micropore volume determined via $\mathrm{N}_{2}$-DFT model. ${ }^{d}$ Determined via EA after sulfonation with $\mathrm{SO}_{3}$. A reference of non-sulfonated CTF contained $<0.01 \mathrm{wt} \%$ of sulfur. ${ }^{e}$ Material obtained via small scale synthesis as described in prior studies. ${ }^{29}$ With S-CTF-b* as reproduction of SCTF-b. 
Generally, the sulfonation of materials can be carried out in the liquid or the gas phase with sulfuric acid or oleum as a sulfonation agent. ${ }^{30-34}$ Prior studies could not confirm the sulfonation of CTF materials in the liquid phase. ${ }^{27}$ Therefore, the sulfonation of the CTFs investigated in the present study was carried out in the gas-phase as it was presented by Richter et al. ${ }^{34}$ using $20 \%$ oleum (20 wt $\%$ of $\mathrm{SO}_{3}$ in sulfuric acid). Applying a dynamic vacuum, $\mathrm{SO}_{3}$ is evaporated from a reservoir, passing through the material exhibited within a glass reactor. The apparatus for the sulfonation is depicted in the ESI (Fig. S8†). The sulfonation in the gas-phase takes place under mild conditions at room temperature and enables easy work-up, while sole adsorption of $\mathrm{H}_{2} \mathrm{SO}_{4}$ from the liquid phase on the materials surface is suppressed.

The sulfonation of all CTF materials leads to a decrease in both porosity as well as specific surface area (Table 1, entries 3 , $6,7,10$ and 13), which cannot only be explained by the increasing weight after introduction of the sulfonic acid group. More likely, the introduction of sterically demanding $-\mathrm{SO}_{3} \mathrm{H}$ groups after sulfonation is responsible for the blockage of the pore system. The course of $\mathrm{N}_{2}$ physisorption isotherms of the sulfonated materials (referred to as S-CTF) shows a comparable trend to the untreated materials, indicating that the porous structure stays intact even after treatment (Fig. S1-S4†). The pore size distribution calculated for all S-CTF materials reveals a loss of porosity mainly in the mesoporous range for bimodal samples, while the micropores are mostly unaffected. These structural findings could be reproduced excellently for all four CTFs (Table S1 and Fig. S7†).

TEM images of a sulfonated S-CTF-d in comparison to untreated CTF-d do not reveal any signs of degradation or decomposition (Fig. 1). Furthermore, SEM/EDX mapping images of S-CTF-d confirm sulfur evenly distributed throughout the whole CTF-d material (Fig. 2).

For all S-CTF samples a similar loading of sulfur around 1.2 to $1.6 \mathrm{wt} \%$ has been determined via elemental analysis (Table 1). Interestingly, S-CTF-b based on the pyridinic 2,6-DCP monomer contains nearly twice the amount of sulfur after sulfonation (2.67 wt\%, Table 1, entry 6). This might be assigned to a $\mathrm{SO}_{3}$-pyridine adduct formation after treatment with $\mathrm{SO}_{3}$ in the gas-phase. Reproducibility tests confirm this assumption, as the reproduced S-CTF-b contained only $0.72 \mathrm{wt} \% \mathrm{~S}$, while the
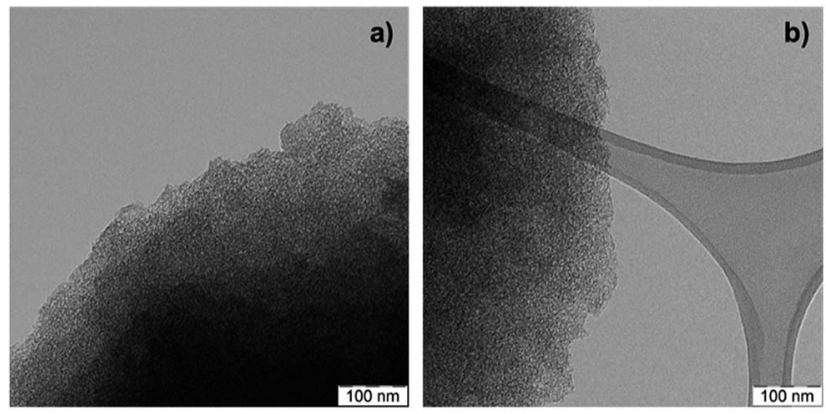

Fig. 1 TEM images of (a) untreated CTF-d and (b) S-CTF-d after sulfonation with $\mathrm{SO}_{3}$ in the gas-phase. other S-CTF materials showed comparable loadings as found for the first experiments after extensive washing with an excess of water for a prolonged time period (Table 1, entry 7).

The acidity of all samples was determined via titration, ICPOES analysis after digestion of the S-CTF and elemental analysis (EA). All analysis techniques give comparable results for the acidity of the S-CTFs being approximately between 0.4 and $0.8 \mathrm{mmol} \mathrm{g}^{-1}$ (Table 2). The results of all the methods were in line with each other. The S-content determined via EA was used to calculate the sulfonation degree for all samples. The degree of sulfonation was calculated for the entire material, assuming complete and ideal polymerization of the corresponding monomer.

In general, the sulfur loading does not follow a particular trend but all S-CTF materials contain around 1.0-1.5 wt\% of sulfur $\left(\sim 0.4 \mathrm{mmol} \mathrm{g}^{-1}\right)$ at a sulfonation degree in-between 5$10 \%$ on average. This could also be shown via reproducibility tests, which confirm only small discrepancies when sulfonating the same CTF type under identical conditions (Table S1†). The degree of sulfonation for CTF materials is relatively low when compared to commercially available solid acids. E.g. the degree of sulfonation for an amberlyst resin with ion-exchange capac-

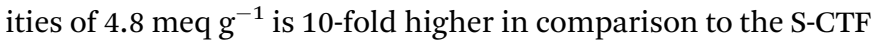
materials presented in the present study. On the one hand, this might reflect the high amount of triazine and pyridine moieties and their electronic properties hindering the sulfonation of every single monomer. Especially the high degree of carbonization leads to a reduction of the aromatic functionalities suitable for sulfonation. Hence, there is no idealized polymer structure available as it is for defined polymers such as polystyrene in the case of Amberlyst ${ }^{\circledR}$ resins. On the other hand, the partially carbonized regions of the CTF materials might have been sulfonated, meaning that the amount of fixated $\mathrm{SO}_{3} \mathrm{H}$ functionalities does not correspond to the ideal aromatic functions within the material, but rather to the expected carbonized units. However, distinction between both functionalizations is rather difficult and even via ${ }^{13} \mathrm{C}-\mathrm{NMR}$ of different sulfonated carbons, ${ }^{20}$ no clear evidence of the incorporated $\mathrm{SO}_{3} \mathrm{H}$-function could be verified.

Thermogravimetric analysis of sulfonated S-CTF-a, -c and -d indicate the loss of the sulfonic acid functionalities at temperatures above $300{ }^{\circ} \mathrm{C}$ (Fig. S5 and S6 $\dagger$ ). Decomposition of the porous material is initiated at temperatures above $400{ }^{\circ} \mathrm{C}$. In the case of CTF-b, desulfonation takes place at somewhat lower temperatures. This can be attributed to the assumed adduct formation to the pyridinic function of the 2,6-DCP monomer.

Additionally, CTF-a was sulfonated in the liquid phase to compare the materials obtained by different procedures (Table S1 and Fig. S7†). Interestingly, the liquid phase sulfonation resulted in higher sulfur loadings of $3.0 \mathrm{wt} \%$ leading to an acidity of $0.96 \mathrm{mmol} \mathrm{g}^{-1}$ and thus a sulfonation degree of approximately $12.2 \%$. The amount of acid sites is 2 -fold higher compared to the gas-phase sulfonation method, but can mostly be attributed to adsorption effects of $\mathrm{H}_{2} \mathrm{SO}_{4}$ on the materials surface and adduct formation to the triazine moieties, which cannot be suppressed in the liquid phase. This assumption can further be confirmed by thermogravimetric analysis as the desulfonation begins at 

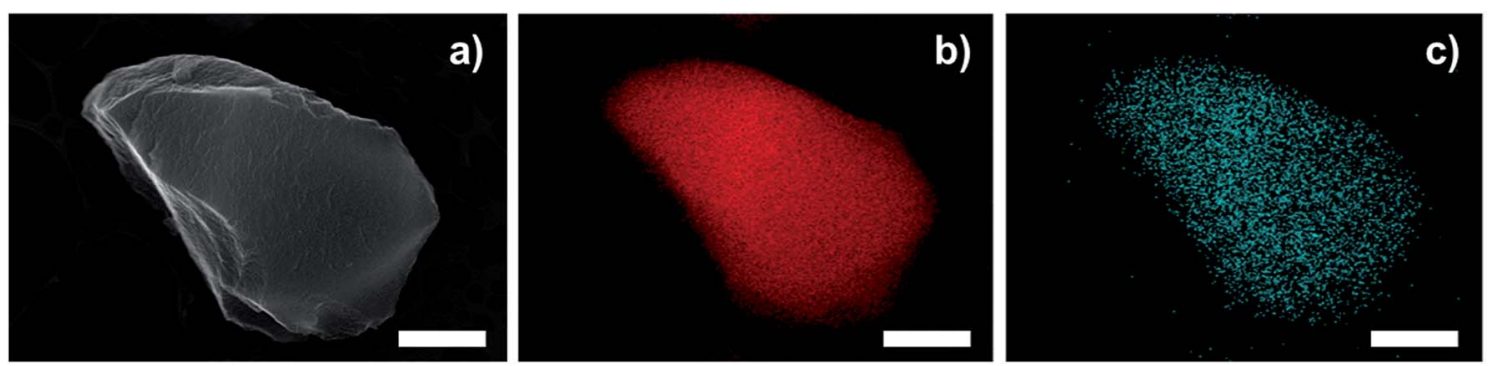

Fig. 2 SEM/EDX mapping images of S-CTF-d. (a) SEM of S-CTF-d particle. (b) C mapping (red). (c) S mapping (blue). The scale bars represent $1 \mu \mathrm{m}$.

Table 2 Acidities of sulfonated CTF materials determined via titration, ICP-OES measurements and elemental analysis to calculate the degree of sulfonation

\begin{tabular}{lllllc}
\hline No. & Material & $\begin{array}{l}\text { Titration }^{a} \\
{\left[\mathrm{mmol} \mathrm{g}^{-1}\right]}\end{array}$ & $\begin{array}{l}\text { ICP-OES }^{b} \\
{\left[\mathrm{mmol} \mathrm{g}^{-1}\right]}\end{array}$ & $\begin{array}{l}\mathrm{EA}^{c} \\
{\left[\mathrm{mmol} \mathrm{g}^{-1}\right]}\end{array}$ & $\begin{array}{l}\text { S-degree }^{d} \\
{[\%]}\end{array}$ \\
\hline 1 & S-CTF-a & 0.38 & 0.36 & 0.37 & 4.7 \\
2 & S-CTF-b & 0.59 & 0.60 & 0.83 & 10.7 \\
3 & S-CTF-b* & - & - & 0.23 & 2.8 \\
4 & S-CTF-c & 0.53 & 0.34 & 0.40 & 5.1 \\
5 & S-CTF-d & 0.61 & 0.46 & 0.49 & 10.1
\end{tabular}

${ }^{a}$ Ion exchange in saturated $\mathrm{NaCl}$ solution followed by titration with $\mathrm{NaOH}(0.005 \mathrm{M}) .{ }^{b}$ Derived from sulfur content determined via ICPOES analysis. ${ }^{c}$ Calculated from sulfur content determined via elemental analysis. ${ }^{d}$ Degree of sulfonation calculated as amount of sulfur (mmol) to amount of monomer $(\mathrm{mmol})$ for entire material assuming complete and ideal polymerization. With S-CTF-b* as reproduction of S-CTF-b.

relatively low temperatures in comparison to the S-CTFa sulfonated in the gas-phase (Fig. S5†).

It is well-known that sulfonated carbons can catalyze the hydrolysis of cellulose and cellobiose to glucose at high conversions and yields. ${ }^{20}$ Since the CTF materials presented in this work can be allocated to sulfonated $\mathrm{N}$-doped carbon systems, we decided to investigate them within the same catalytic reaction in order to verify their successful functionalization.

\section{Catalytic hydrolysis of cellobiose to glucose}

The synthesized S-CTF materials were tested for the hydrolysis of $10 \mathrm{wt} \%$ aqueous cellobiose solution at $150{ }^{\circ} \mathrm{C}$. The results of the tests are shown in Table 3 . All the sulfonated materials were catalytically active for the hydrolysis. Very high cellobiose conversions up to $88 \%$ are attainable over mesoporous catalysts, whereas only $31-55 \%$ were reached over microporous catalysts for $2 \mathrm{~h}$ of reaction (Table 3 , entry 5 and 11). Prolongation of the reaction time for another $2 \mathrm{~h}$ did not bring a significant increase of the conversion (Table 3, entry 6 and 12). This points at deactivation of the microporous catalysts under the reaction conditions, probably due to adsorption of substrate/product in the micropores. Additionally, leaching of sulfur corresponding to $20-40 \%$ of its initial content in the materials was observed (Table 3). Leaching of sulfonic groups into the aqueous medium led to formation of sulfuric acid under reaction conditions. As a result, acidification took place during the experiments, and slightly acidic solutions were obtained after filtration of the reaction mixtures.

Hydrolysis of cellobiose can proceed both homogeneously and heterogeneously catalyzed, as shown in Fig. 3. The first orders on cellobiose and $\mathrm{H}^{+}$active species were assumed. ${ }^{37}$ The parallel processes yield glucose with the rate constants of hydrolysis $k_{\text {homo }}$ and $k_{\text {hetero }}$, respectively. Apparently, the overall rate constant for the hydrolysis can be expressed as $k_{\mathrm{hydr}}=$

Table 3 Results of catalytic tests of S-CTF catalysts for hydrolysis of cellobiose ${ }^{a}$

\begin{tabular}{|c|c|c|c|c|c|c|c|c|c|}
\hline No. & Catalyst & $t[\mathrm{~h}]$ & $X[\%]$ & $Y[\%]$ & $\mathrm{pH}$ & Leached sulfur $[\%]$ & $k_{\text {hydr }}\left[\mathrm{h}^{-1}\right]$ & $k_{\text {homo }}\left[\mathrm{H}^{+}\right] / k_{\text {hydr }}[\%]$ & $k_{\text {hetero }}\left[\mathrm{L} \mathrm{mol}^{-1} \mathrm{~h}^{-1}\right]$ \\
\hline 1 & S-CTF-a & 1 & 32.9 & 33.3 & 2.5 & 34.0 & 0.58 & 53 & 7.2 \\
\hline 2 & S-CTF-a & 2 & 59.2 & 59.7 & 2.5 & 42.4 & & & \\
\hline 3 & S-CTF-a & 4 & 88.1 & 85.6 & 2.2 & 48.4 & & & \\
\hline 4 & S-CTF-b & 1 & 3.4 & 10.9 & 3.3 & 38.1 & 0.33 & 24 & 5.0 \\
\hline 5 & S-CTF-b & 2 & 30.6 & 24.7 & 3.2 & 43.8 & & & \\
\hline 6 & S-CTF-b & 4 & 34.6 & 27.8 & 3.2 & 29.8 & & & \\
\hline 7 & S-CTF-c & 1 & 41.3 & 42.7 & 2.7 & 29.5 & 0.62 & 44 & 8.6 \\
\hline 8 & S-CTF-c & 2 & 49.6 & 51.5 & 2.6 & 24.7 & & & \\
\hline 9 & S-CTF-c & 4 & 85.4 & 83.9 & 2.6 & 46.2 & & & \\
\hline 10 & S-CTF-d & 1 & 20.0 & 23.5 & 2.7 & 27.8 & 0.59 & 46 & 6.5 \\
\hline 11 & S-CTF-d & 2 & 55.8 & 62.3 & 2.6 & 32.0 & & & \\
\hline 12 & S-CTF-d & 4 & 66.2 & 66.0 & 2.6 & 32.9 & & & \\
\hline
\end{tabular}

${ }^{a}$ Reaction conditions: $1-4 \mathrm{~h}, 150{ }^{\circ} \mathrm{C}, 30$ bar of $\mathrm{N}_{2}$ at $750 \mathrm{rpm}$. Acid : cellobiose molar ratio (given in brackets): S-CTF-a (1.7 mol\%), S-CTFb (0.7 mol\%), S-CTF-c (1.8 mol\%) and S-CTF-d (1.4 mol\%). 


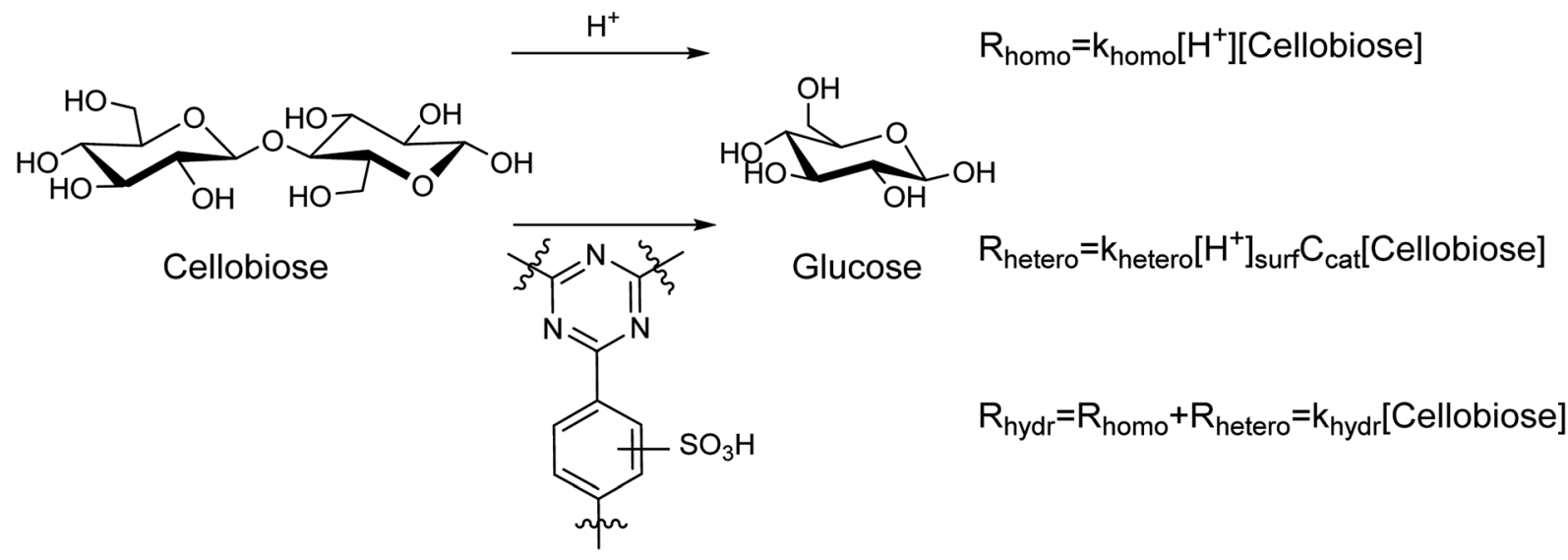

Fig. 3 Parallel homogeneous and heterogeneous routs for cellobiose hydrolysis in the presence of S-CTF. $R_{\text {homo, }} R_{\text {hetero, }}$ and $R_{\text {hydr }}$ denote the rate of homogeneous hydrolysis, the rate of heterogeneous hydrolysis, and the apparent reaction rate $\left(\mathrm{mol} \mathrm{L}^{-1} \mathrm{~h}^{-1}\right)$. The rate constants of homogeneous ( $k_{\text {homo, }} \mathrm{L} \mathrm{mol}^{-1} \mathrm{~h}^{-1}$ ) and heterogeneous $\left(k_{\text {hetero, }} \mathrm{L} \mathrm{mol}^{-1} \mathrm{~h}^{-1}\right)$ hydrolysis as well as overall rate constant $k_{\text {hydr }}\left(\mathrm{h}^{-1}\right)$ are considered, $\left[\mathrm{H}^{+}\right]$and [cellobiose] denote concentrations $\left(\mathrm{mol} \mathrm{L}^{-1}\right)$ of these species in aqueous phase, $\mathrm{C}_{\text {cat }}$ is concentration of the catalyst $\left(\mathrm{g} \mathrm{L}^{-1}\right)$. $\left[\mathrm{H}^{+}\right]_{\text {surf }}$ is a surface concentration of acidic groups $\left(\mathrm{mol} \mathrm{g}^{-1}\right)$.

$\left(k_{\text {homo }}\left[\mathrm{H}^{+}\right]+k_{\text {hetero }}\left[\mathrm{H}^{+}\right]_{\text {surf }} C_{\text {cat }}\right)$. The values of $k_{\text {hydr }}$ were obtained from the results of cellobiose hydrolysis in the presence of the sulfonated CTF materials (Table 3).

Homogeneous hydrolysis of cellobiose under the applied conditions was investigated. A blank test of cellobiose hydrolysis demonstrated that hydrolysis takes place even in the absence of a catalyst. The hydrolysis of cellobiose accelerated during the course of the experiment (Fig. S9†). This is in line with acidification of the aqueous medium from $\mathrm{pH} 5.2$ till $\mathrm{pH} 3$ for the first hour of the hydrolysis. Further prolongation of the reaction did not lead to dramatic change of the $\mathrm{pH}$ value (Fig. S9†). The observed $\mathrm{pH}$ change can be explained by formation of acidic by-products upon dehydration of saccharides. It must be noted that the reaction mixture turned brownish upon hydrolysis in pure water. Clearly, despite rather high selectivity for glucose (88-95\%), the saccharides partly underwent degradation forming humins. Next, $\mathrm{H}_{2} \mathrm{SO}_{4}$ was tested for hydrolysis of cellobiose. In these tests, the concentration of sulfuric acid ( $c a .1 .6 \mathrm{mM}, \mathrm{pH} 2.4$ ) was close to that obtained over S-CTFs due to leaching of sulfur (Table 3). Again, darkening of the reaction solution took place owing to formation of humins. Finally, we studied catalytic activity of nonsulfonated CTF-a and a mixture of CTF-a with $\mathrm{H}_{2} \mathrm{SO}_{4}$. Interestingly, filtration of the reaction mixture after the experiments gave transparent solutions. This indicates efficient adsorption of humins onto CTF under the reaction conditions. Furanic compounds, mainly HMF, are products of consecutive

Table 4 Estimated rate constants of homogeneous cellobiose hydrolysis

\begin{tabular}{lll}
\hline No. & Catalyst & $k_{\mathrm{homo}}, \mathrm{L} \mathrm{mol}^{-1} \mathrm{~h}^{-1}$ \\
\hline 1 & $\mathrm{No}$ & 134 \\
2 & $\mathrm{H}_{2} \mathrm{SO}_{4}$ & 117 \\
3 & $\mathrm{CTF}^{-a}$ & 127 \\
4 & $\mathrm{H}_{2} \mathrm{SO}_{4}+\mathrm{CTF}-\mathrm{a}$ & 114
\end{tabular}

dehydration of glucose under acidic conditions. A generally accepted mechanism of humin formation comprehends condensation of the furanics with saccharides. ${ }^{38}$ Apparently, adsorption of humins onto CTF is favorable due to high affinity of HMF towards aromatic functionalities of CTF. ${ }^{39}$ Furthermore, the $\mathrm{pH}$ value of the reaction mixture after the reaction over CTF $+\mathrm{H}_{2} \mathrm{SO}_{4}$ (2.9) was slightly higher compared to the $\mathrm{pH}_{0}$ value of 2.4. This can be explained by adsorption of sulfuric acid onto basic centers of the CTF material. We performed a control experiment to investigate adsorption of $\mathrm{H}_{2} \mathrm{SO}_{4}$ onto CTF at room temperature. Stirring of $1.6 \mathrm{mM} \mathrm{H}_{2} \mathrm{SO}_{4}$ with CTF-a led to total neutralization of the solution, apparently due to interaction of the strong acid with nitrogen of triazine rings (Fig. S10 $\dagger$ ). The values of $k_{\text {homo }}$ were estimated based on the obtained data (Table 4). Interestingly, the rate constants appear to be similar for different reaction conditions and independent of the nature of acid $\left(\mathrm{H}_{2} \mathrm{SO}_{4}\right.$ or organic acids produced due to dehydration of saccharides). The presence of the CTF-a material does not influence the rate constant of cellobiose hydrolysis. Data on $\mathrm{pH}$ of reaction mixture (Table 3 ), overall hydrolysis constants $k_{\text {hydr }}$ (Table 3), concentration of surface acidic sites (Table 2), and $k_{\text {homo }}\left(c a .123 \mathrm{~L} \mathrm{~mol}^{-1} \mathrm{~h}^{-1}\right.$, according to the data from Table 4) enable estimation of $k_{\text {hetero }}$ values according to the equations presented in Fig. 3. The results of these calculations are listed in Table 3, indicating that the homogeneous reaction makes a contribution of $24-53 \%$ to the apparent rate of cellobiose hydrolysis. The apparent $k_{\text {hetero }}$ appeared to be similar for all the materials, though a somewhat lower value was observed for CTF-b.

Activation energy of the hydrolysis equals $c a .115 \mathrm{~kJ} \mathrm{~mol}^{-1}$ in the presence of the most active catalyst S-CTF-a (Fig. S11 $\dagger$ ), similar to the previously obtained data for molecular acids. ${ }^{37,40}$ Importantly, share of the leached out sulfur of $\mathrm{ca}$. 20\% does not dramatically depend on the reaction temperature (Fig. 4). This indicates the presence of weakly bound sulfur-containing groups, which are easily removed upon contact with hot 

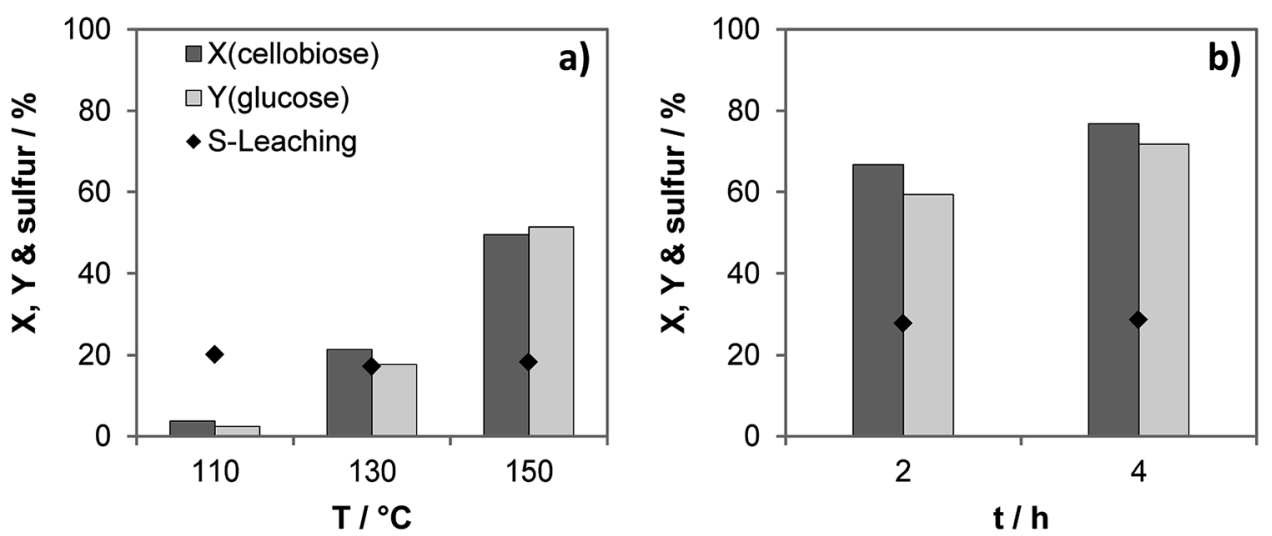

Fig. 4 (a) Temperature dependency of the cellobiose conversion $(X)$, glucose yield $(Y$ ) and sulfur leaching; (b) hot filtration test. Reaction conditions: $2 \mathrm{~h}, 110-150{ }^{\circ} \mathrm{C}, 30$ bar of $\mathrm{N}_{2}$ at $750 \mathrm{rpm}, 5 \mathrm{~mL}$ of $10 \mathrm{wt} \%$ cellobiose, $50 \mathrm{mg}$ of S-CTF-a.

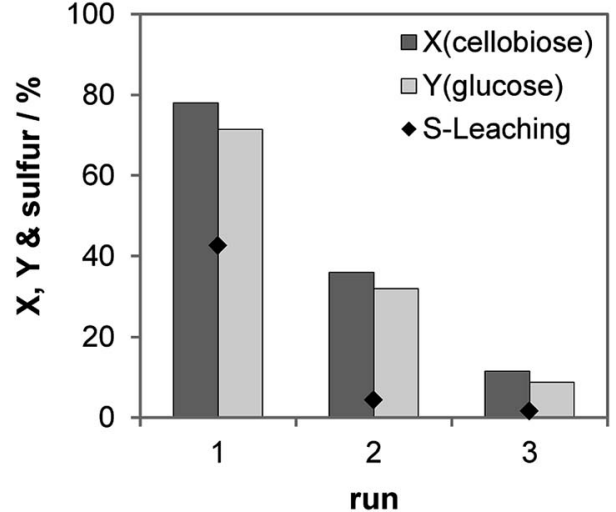

Fig. 5 Recycling study of S-CTF-a. Reaction conditions: $2 \mathrm{~h}, 150{ }^{\circ} \mathrm{C}$ 30 bar of $\mathrm{N}_{2}$ at $750 \mathrm{rpm}, 5 \mathrm{~mL} 10 \mathrm{wt} \%$ cellobiose, $75 \mathrm{mg} \mathrm{S-CTF-a}$

water. Previous investigations suggest that sulfonated carbon materials also exhibit weakly- and strongly bound sulfonic groups. ${ }^{41,42}$ Pre-treatment of the sulfonated carbons with hot water results in removal of the weakly bound sulfur, whereas strongly bound groups were stable under the reaction conditions of cellobiose hydrolysis. ${ }^{41,43}$ This was also indicated via a hot filtration test resulting in only slight increase of conversion and yield after filtration of the solid catalyst and processing the liquid phase for additional 2 hours (Fig. 4b).

We studied the recycling of the S-CTF-a catalyst (Fig. 5). As expected, high amounts of sulfur, corresponding to $43 \%$ content in solid catalyst, were only leached in the first run. In the following two runs, only 4 and $2 \%$ of the initial S concentration were detected in the product solution. In total, $c a .49 \%$ of the initial sulfur content was leached out of the catalyst during the three consecutive runs, which was confirmed by ICPOES analysis of the liquid after reaction and the elemental analysis of the used catalyst. A significant drop of the cellobiose conversion from 78 to $36 \%$ was observed for the first and the second run, which can be explained by considerable loss of sulfur during the first run. Despite very low leaching of sulfur during the second and the third runs, further decrease of the cellobiose conversion to $12 \%$ occurred in the third run. Therefore, catalyst deactivation cannot only be attributed to the leaching of the catalytically active species. Our results on molecular $\mathrm{H}_{2} \mathrm{SO}_{4}$ physically mixed with CTF-a (see above) suggest that the materials are very prone to adsorption of partly dehydrated by-products, which probably brings about blockage of active sites and drop of catalytic activity.

Further improvements of the catalytic activity can possibly be achieved by optimization of the procedure of catalyst synthesis and pretreatment, ${ }^{41,42}$ as well as conducting the reaction under continuous conditions to facilitate the removal of (by)-products from the reactor. ${ }^{43,44}$

\section{Conclusions}

Sulfonation of CTF materials was conducted for the first time by treating the materials with gas-phase $\mathrm{SO}_{3}$. This procedure was successfully applied to obtain four S-CTFs which vary in composition of the monomers. Sulfonation degrees in the range of $5-10 \%$ and an acid surface concentration of around 0.4$0.8 \mathrm{mmol} \mathrm{g}^{-1}$ were reached. The acid sites are evenly distributed within the CTF materials, while the material itself does not show any signs of decomposition after sulfonation. Thermal stability of the sulfonated S-CTFs suggests a covalent binding of the introduced acid sites. Notably, the CTFs preserve their structural properties and slight losses in specific surface area as well as porosity can be assigned mostly to the space demanding acid sites.

Catalytic testing provides evidence of the successful acid functionalization of the polymers. The S-CTF materials were active when applied in the catalytic hydrolysis of cellobiose to glucose yielding up to $86 \%$ glucose in 4 hours at a rather high substrate-to-catalyst weight ratio of 10 . Very high selectivity of glucose formation over $90 \%$ was observed for hydrolysis in the presence of S-CTF. Leaching of sulfur took place during the course of hydrolysis, as it is expected for any sulfonated materials under the hydrothermal reaction conditions. Additionally, co-formation of acidic by-products was observed. $\mathrm{Ca}$. $24-53 \%$ of cellobiose were hydrolyzed over S-CTFs due to contribution of 
homogeneous catalysis. The leaching along with strong adsorption of (by)-products on the surface of the S-CTF appears to be a reason for catalyst deactivation. Nevertheless, the successful sulfonation of CTFs in combination with afore published coordination approaches of molecular catalysts within the frameworks enables new synthesis routes for multifunctional catalysts. In this manner, the design of a catalyst containing basic coordination sites for molecular or nanoparticulate species, tunable structural parameters as well as surface acidity opens the path to numerous applications, enabling the rational development of multi-step reactions at one sole catalyst system.

\section{Conflicts of interest}

There are no conflicts to declare.

\section{Acknowledgements}

We acknowledge financial support from the DFG (PA1689/1-1) and funding from the Excellence Initiative of the German federal and state governments. SEM/EDX mapping and TEM imaging was performed at the Center for Chemical Polymer Technology CPT, which is supported by the EU and the federal state of North Rhine-Westphalia (grant no. EFRE 3000883 02).

\section{Notes and references}

1 M. Baerns, A. Behr, A. Brehm, J. Gmehling, H. Hofmann, U. Onken and A. Resch, in Technische Chemie, Wiley-VCH, Weinheim, 1st edn, 2006.

2 M. B. Hocking, in Handbook of Chemical Technology and Pollution Control, Academic Press, London, 2nd edn, 1998.

3 A. Corma, Chem. Rev., 1995, 95, 559-614.

4 K. Tanabe and W. F. Hölderich, Appl. Catal., A, 1999, 181, 399-434.

5 A. Corma and H. Garciam, Catal. Today, 1997, 38, 257-308.

6 S. M. Auerbach, K. A. Carrado and P. K. Dutta, in Handbook of Zeolithe Science and Technology, Marcel Dekker Inc., Basel, 1st edn, 2003.

7 T. Okuhara, Chem. Rev., 2002, 102, 3641-3666.

8 M. A. Harmer and Q. Sun, Appl. Catal., A, 2001, 221, 45-62.

9 V. V. Ordomsky, J. C. Schouten, J. van der Schaaf and

T. A. Nijhuis, Chem. Eng. J., 2012, 207, 218-255.

10 Dow Chemical Company, Form No. 177-02436-0511, 2011.

11 J. H. Clark, Acc. Chem. Res., 2002, 35, 791-797.

12 E. Lotero, D. E. Lopez, K. Suwannakarn, D. A. Bruce and J. G. Goodwin Jr, Ind. Eng. Chem. Res., 2005, 44, 5353-5363.

13 P. A. Russo, M. M. Antunes, P. Neves, P. V. Wiper, E. Fazio,

F. Neri, F. Barreca, L. Mafra, M. Pillinger, N. Pinna and

A. A. Valente, J. Mater. Chem. A, 2014, 2, 11813-11824.

14 S. Van de Vyver, J. Geboers, P. A. Jacobs and B. F. Sels, ChemCatChem, 2011, 3, 82-94.

15 P. Lanzafame, D. M. Temi, S. Perathoner, G. Centi, A. Macario, A. Aloise and G. Giordano, Catal. Today, 2011, 175, 435-441.
16 (a) J.-P. Lange, Angew. Chem., Int. Ed., 2015, 54, 13186-13197; (b) A. T. To, P.-W. Chung and A. Katz, Angew. Chem., Int. Ed., 2015, 54, 11050-11053.

17 L. Vilcocq, P. C. Castilho, F. Carvalheiro and L. C. Duarte, ChemSusChem, 2014, 7, 1010-1019.

18 H. Wang, Y. Guo, C. Chang, X. Zhu, X. Liu, J. Han and Q. Ge, Appl. Catal., A, 2016, 523, 182-192.

19 (a) Y. Kim, R. Hendrickson, N. Mosier and M. R. Ladisch, Energy Fuels, 2005, 19, 2189-2200; (b) A. Takagaki, C. Tagusagawa and K. Domen, Chem. Commun., 2008, 5363-5365; (c) L. Shuai and X. Pan, Energy Environ. Sci., 2012, 5, 6889-6894.

20 (a) S. Suganuma, K. Nakajima, M. Kitano, D. Yamaguchi, H. Kato, S. Hayashi and M. Hara, J. Am. Chem. Soc., 2008, 130, 12787-12793; (b) M. Kitano, D. Yamaguchi, S. Suganuma, K. Nakajima, H. Kato, S. Hayashi and M. Hara, Langmuir, 2009, 25, 5068-5075; (c) G. S. Foo, A. H. Van Pelt, D. Krötschel, B. F. Sauk, A. K. Rogers, C. R. Jolly, M. M. Yung and C. Sievers, ACS Sustainable Chem. Eng., 2015, 3, 1934-1942.

21 J. A. Bootsma and B. H. Shanks, Appl. Catal., A, 2007, 327, 4451.

22 (a) M. Karaki, A. Karout, J. Toufaily, F. Rataboul, N. Essayem and B. Lebeau, J. Catal. , 2013, 305, 204-216; (b) L. Sandbrink, T. Lazaridis, M. Rose and R. Palkovits, Microporous Mesoporous Mater., 2018, 267, 198-202.

23 R. Ormsby, J. R. Kastner and J. Miller, Catal. Today, 2012, 190, 89-97.

24 X. Zhao, J. Wang, C. Chen, Y. Huang, A. Wang and T. Zhang, Chem. Commun., 2014, 50, 3439-3442.

25 (a) P. Kuhn, M. Antonietti and A. Thomas, Angew. Chem., Int. Ed., 2008, 47, 3450-3453; (b) P. Kuhn, A. Thomas and M. Antonietti, Macromolecules, 2009, 42, 319-326.

26 (a) J. Roeser, K. Kailasam and A. Thomas, ChemSusChem, 2012, 5, 1793-1799; (b) P. Katekomol, J. Roeser, M. Bojdys, J. Weber and A. Thomas, Chem. Mater., 2013, 25, 1542-1548.

27 (a) R. Palkovits, M. Antonietti, P. Kuhn, A. Thomas and F. Schüth, Angew. Chem., Int. Ed., 2009, 48, 6909-6912; (b) M. Soorholtz, R. J. White, T. Zimmermann, M.-M. Titirici, M. Antonietti, R. Palkovits and F. Schüth, Chem. Commun., 2013, 49, 240-242.

28 (a) C. E. Chan-Thaw, A. Villa, G. M. Veith, K. Kailasam, L. A. Adamczyk, R. R. Unocic, L. Prati and A. Thomas, Chem.-Asian J., 2012, 7, 387-393; (b) S. Armenise, L. Roldán, Y. Marco, A. Monzón and E. García-Bordejé, J. Phys. Chem. C, 2012, 116, 26385-26395.

29 (a) J. Artz, S. Mallmann and R. Palkovits, ChemSusChem, 2015, 8, 672-679; (b) J. Artz and R. Palkovits, ChemSusChem, 2015, 8, 3832-3838; (c) A. K. Beine, A. J. D. Krüger, J. Artz, C. Weidenthaler, C. Glotzbach, P. J. C. Hausoul and R. Palkovits, Green Chem., 2018, 20, 1316-1322; (d) J. Artz, ChemCatChem, 2018, 10, 1753-1771.

30 R. Brückner, in Reaktionsmechanismen, Spektrum Akademischer Verlag, Berlin, 3rd edn, 2007.

31 F. Kucera and J. Jancar, Polym. Eng. Sci., 1998, 38, 783-792.

32 F. H. Richter, K. Pupovac, R. Palkovits and F. Schüth, ACS Catal., 2013, 3, 123-127. 
33 G. Schäfer, P. Neumann and G. Blinne, German patent, DE3732539 A1, BASF, 1989.

34 F. H. Richter, Y. Meng, T. Klasen, L. Sahraoui and F. Schüth, J. Catal., 2013, 308, 341-351.

35 C. Bai, M. Zhang, B. Li, Y. Tian, S. Zhang, X. Zhao, Y. Li, L. Wang, L. Ma and S. Li, J. Hazard. Mater., 2015, 300, 368377.

36 S. N. Talapaneni, T. H. Hwang, S. H. Je, O. Buyukcakir, J. W. Choi and A. Coskun, Angew. Chem., Int. Ed., 2016, 55, 3106-3111.

37 (a) O. Bobleter, W. Schwald, R. Concin and H. Binder, J. Carbohydr. Chem., 1986, 5, 387-399; (b) N. S. Mosier, C. M. Ladisch and M. R. Ladisch, Biotechnol. Bioeng., 2002, 79, 610-618.

38 (a) M. Sevilla and A. B. Fuertes, Carbon, 2009, 47, 2281; (b) M. Sevilla and A. B. Fuertes, Chem.-Eur. J., 2009, 15, 41954203; (c) I. van Zandvoort, Y. Wang, C. B. Rasrenda, E. R. H. van Eck, P. C. A. Bruijnincx, H. J. Heeres and B. M. Weckhuysen, ChemSusChem, 2013, 6, 1745-1758.
39 C. Detoni, C. H. Gierlich, M. Rose and R. Palkovits, ACS Sustainable Chem. Eng., 2014, 2, 2407-2415.

40 L. Negahdar, J. U. Oltmanns, S. Palkovits and R. Palkovits, Appl. Catal., B, 2014, 147, 677-683.

41 (a) A. Onda, T. Ochi and K. Yanagisawa, Green Chem., 2008, 10, 1033-1037; (b) M. Karaki, A. Karout, J. Toufaily, F. Rataboul, N. Essayem and B. Lebeau, J. Catal., 2013, 305, 204-216.

42 A. H. Van Pelt., O. A. Simakova, S. M. Schwimming, J. L. Ewbank, G. S. Foo, E. A. Pidko, E. J. M. Hensen and C. Sievers, Carbon, 2014, 77, 143-154.

43 G. S. Foo, A. H. Van Pelt, D. Krötschel, B. F. Sauk, A. K. Rogers, C. R. Jolly, M. M. Yung and C. Sievers, ACS Sustainable Chem. Eng., 2015, 3, 1934-1942.

44 I. Delidovich and R. Palkovits, Catal. Sci. Technol., 2014, 4, 4322-4329.

45 I. Delidovich and R. Palkovits, Microporous Mesoporous Mater., 2016, 219, 317-321. 